\title{
Médiévales
}

Langues, Textes, Histoire

70 | printemps 2016

Lieux d'hygiène et lieux d'aisance en terre d'Islam (VII $\mathrm{e}_{-}$ $\mathrm{XV}^{\mathrm{e}}$ siècle)

\section{L'examen des urines dans la médecine médiévale en terre d'Islam et en Occident. Un aperçu}

The Examination of Urine in Medieval Medicine, between Islamic and Western Worlds. A Short Overview

\section{Laurence Moulinier-Brogi}

\section{OpenEdition \\ Journals}

Édition électronique

URL : http://journals.openedition.org/medievales/7707

DOI : $10.4000 /$ medievales. 7707

ISSN : 1777-5892

Éditeur

Presses universitaires de Vincennes

Édition imprimée

Date de publication : 23 juin 2016

Pagination : 25-41

ISSN : 0751-2708

Référence électronique

Laurence Moulinier-Brogi, «L'examen des urines dans la médecine médiévale en terre d'Islam et en Occident. Un aperçu », Médiévales [En ligne], 70 I printemps 2016, mis en ligne le 15 juin 2018, consulté le 07 mai 2019. URL : http://journals.openedition.org/medievales/7707 ; DOI : 10.4000/ medievales.7707 
Laurence Moulinier-Brogi

\section{L'examen des urines dans la médecine médiévale en terre d'Islam et en Occident}

Un aperçu

Le sujet de cette contribution est sinon périphérique du moins dans une relation synecdotique avec le thème du dossier ici réuni. Je m'attacherai en effet non à des lieux d'aisance, mais au produit d'une évacuation corporelle et son contenant, à savoir l'urine et le flacon dans lequel elle pouvait être recueillie pour l'examen clinique d'un patient : c'est l'urinal, dans lequel étaient collectées les urines sur l'inspection desquelles se fondait le diagnostic comme le pronostic.

Depuis la haute Antiquité, on estimait que les fluides circulant à l'intérieur du corps et pouvant en sortir transmettaient des informations sur l'état de santé pour qui savait les lire : la peau était vue de très longue date comme renfermant un monde invisible dont, pour les herméneutes, certaines manifestations sensibles traduisaient les mécanismes. Les premiers exemples d'inspection des urines seraient fournis par la civilisation babylonienne, il y a quelque 6000 ans $^{1}$; les médecins indiens auraient été, pour leur part, capables d'en distinguer dix types pathologiques, et l'interprétation des urines avait cours aussi dans l'Égypte ancienne, en particulier pour permettre de diagnostiquer une grossesse ${ }^{2}$.

D'une part, cependant, l'inspection des urines n'était pas universellement pratiquée. Au XIII e siècle, le franciscain Guillaume de Rubrouck, dans sa relation du voyage qu'il fit en Orient entre 1253 et 1255 (Itinerarium ad partes orientales), décrit ainsi les habitants de Cathay : « Leurs médecins connaissent bien la vertu des herbes et les maladies par les pulsations du pouls, mais ils n'ont aucune connaissance des urines ; c'est du moins ce que

1. M. H. HABer, " Pisse Prophecy : A Brief History of Urinalysis », Clinics in Laboratory Medicine, 8 (1988), p. 415-430 (p. 417).

2. H. Christoffel, " Grundzüge der Uroskopie », Gesnerus, 10 (1953), p. 89-122 (p. 89-90). 
j'ai remarqué ${ }^{3}$. » D'autre part, elle n'était à l'origine qu'un des éléments de l'examen clinique, comme le soulignait Hippocrate :

Quant à la médecine [...], ne pouvant faire d'observations directes avec les yeux [...], elle a inventé d'autres ressources auxiliaires : considérant la clarté et la rudesse de la voix, la rapidité et la lenteur de la respiration, et, pour chacun des flux qu'on voit chaque jour, d'une part, la voie par laquelle ils s'échappent, d'une autre, ce qui tient à leur odeur, à leur couleur, à leur degré de ténuité ou d'épaisseur, elle pèse toutes ces circonstances, et juge d'où ils viennent et de quelles parties souffrantes ou pouvant le devenir, elles sont signes ${ }^{4}$.

Or, au cours du Moyen Âge, la sémiologie médicale va se distinguer de celle qui avait cours dans l'Antiquité par un rôle plus important accordé à cet examen des urines : pour le dire très brièvement, jusqu'alors en effet, si celui-ci s'avérait utile en cas de pathologie fébrile, il n'avait pour autre emploi répandu que le domaine de l'urologie. L'urine était un signe parmi d'autres et son inspection était incluse dans une sémiotique générale. La nouveauté vint de Byzance, avec Théophile, que l'on connaît mal et que l'on situe soit au $\mathrm{VII}^{\mathrm{e}}$ soit au $\mathrm{IX}^{\mathrm{e}}$ siècle. Il composa un Peri ouron destiné à combler les lacunes de ses prédécesseurs en ce domaine ${ }^{5}$; ce traité eut une grande influence sur la médecine byzantine et arabe, et l'uroscopie devint une méthode de diagnostic cruciale, comme le montre à l'envi le traité Des urines composé au $\mathrm{X}^{\mathrm{e}}$ siècle par le médecin kairouannais Isaac Israëli (m. v. 955). Le Peri ouron de Théophile et le De urinis d'Isaac, tous deux traduits en latin au $\mathrm{XI}^{\mathrm{e}}$ siècle, eurent un fort impact sur la médecine occidentale, et à la part croissante accordée à l'examen des urines ou uroscopie correspondirent assez vite de nouvelles modalités de l'examen clinique. De fait, dans le triangle formé par le malade, la maladie et le médecin dans l'Antiquité ${ }^{6}$, au Moyen Âge un objet, l'urinal, s'était véritablement immiscé et cela d'autant plus qu'en Occident, le patient pouvait ne pas être physiquement présent lors de la consultation : il était alors représenté à la fois par le vase contenant ses

3. Guillaume de Rubrouck, ambassadeur de saint Louis en Orient. Récit de son voyage, traduit de l'original latin et annoté par Louis de Backer, Paris, 1877, p. 127. Sur la médecine chinoise au Moyen Âge, on verra Médecine, religion et société dans la Chine médiévale, éd. C. DespeuX, Paris, 2010, notamment E. Hsu, « Le diagnostic du pouls dans la Chine médiévale d'après les manuscrits de Dunhuang », p. 107-184.

4. HipPocrate, De l'Art, dans CEuvres choisies, éd. Ch. DAREMBERG, Paris, 1855, 2éd., XII, p. 36-37. Voir aussi HiPPOCRATE, De l'art médical, trad. É. LiTTRÉ, éd. D. GOUREVITCH, préf. D. Gourevitch, M. D. GrMeK et P. PELlegrin, Paris, 1994.

5. Voir récemment à ce sujet $I l$ De urinis di Teofilo Protospatario. Centralità di un segno clinico, éd. L. R. Angeletti, B. CAVArRa et V. GaZZANigA, Rome, 2009.

6. Cf. D. GOUREVITCH, Le Triangle hippocratique dans le monde gréco-romain : le malade, sa maladie et son médecin, Rome, 1984. 
urines, pars pro toto, et par le porteur de l'objet. Tester la valeur du médecin pouvait donc être une tentation des patients qui, parfois, substituaient dans ce but une urine à une autre. C'est ainsi que les modalités de l'examen laissèrent également une place à la duperie et qu'autour de l'urinal put se jouer aussi un rapport de force entre patient et médecin. On tentera donc ici de comparer l'importance de l'examen des urines en Orient et en Occident, et les brèches qu'un tel examen pouvait ouvrir dans la relation de confiance entre malades et praticiens.

\section{L'inspection des urines dans le monde arabe}

L'uroscopie avait été introduite dans le monde islamique par des textes grecs et romains qui y avaient été recueillis, conservés, puis traduits en arabe, souvent après une version intermédiaire syriaque. Elle représente assurément une branche importante de la sémiologie médicale dans la médecine arabe, à la fois théorisée par de grands noms et demandée par les malades, si l'on en croit par exemple al-Rāzī qui raconte, non sans humour, que son prestige diminua dès qu'il ne se contenta plus d'examiner l'urine des malades et d'en déduire leurs faits et gestes, comme tant de charlatans s'en prétendaient capables, mais qu'il entreprit de les interroger longuement ${ }^{7}$.

Sans lui avoir consacré d'œuvre particulière, al-Rāzī (Muhammad ibn Zakarīyyā al-Rāzī, 865-925) et Avicenne (Ibn Sīnā, 980-1037) sont en effet deux grands noms de la médecine arabe attachés à la naissance et à la formation de l'uroscopie en terres d'Islam. Al-Rāzī reconnaissait, dans son Kitāb al-Haw̄̄ (Le livre complet sur la médecine) l'apport de Galien, mais pensait avoir accompli davantage que lui dans l'examen des urines : il se montrait capable notamment de déduire de leur analyse le comportement et le style de vie du patient, comme le montrent aussi les observations cliniques qu'on a conservées de lui ${ }^{8}$. Et dans son Guide du médecin nomade, il ne consacre pas moins de quinze de ses aphorismes («al-Mur $\underline{\text { hhid } ») ~ a ̀ ~ l ' u r i n e . ~}$ Quant à Avicenne, autre Persan qui écrivit lui aussi en arabe (même si l'on a de lui une Épitre sur le pouls en persan), son monumental Canon consacre une très large place à la science des urines, aux différents aspects du fluide, à l'heure à laquelle il doit être recueilli, aux critères auxquels l'urinal doit satisfaire, etc.

Mais c'est surtout à Isaac Israëli, appelé aussi Isaac Judaeus en Occident, qu'il faut faire une place de ce point de vue : né dans une famille juive d'Égypte, sans doute praticien de second ordre à Fusțāț, il

7. D. JACQUART et F. MicheAU, La Médecine arabe et l'Occident médiéval, Paris, 1990, p. 68.

8. Voir M. MEYERHOF, « Thirty-Three Clinical Cases by Rhazes (circa 900 A.D.) », Isis, 23 (1935), p. 321-356. 
commença véritablement sa carrière en devenant disciple d'Ishāq ibn 'Imrān, un médecin musulman venu de Bagdad à la cour aghlabide, avant de servir le fondateur de la dynastie fatimide en Ifrīqiya. Malgré son origine juive, Isaac appartient pleinement à l'histoire de la médecine arabe, à la fois par la langue et par la tradition intellectuelle qu'il adopta, à savoir le galénisme arabe tel qu'il avait été inauguré à Bagdad par Hunayn ibn Ishāq au IX ${ }^{\mathrm{e}}$ siècle $^{9}$ et repris au siècle suivant par 'Alī ibn al-'Abbās al-Madjūsī (Haly Abbas) et Ibn al-Djazzār ${ }^{10}$, et l'importance de ses ouvrages de médecine tient à leur diffusion ultérieure dans le monde tant musulman que chrétien ${ }^{11}$. Son traité sur les urines en particulier, qui présentait la nature du fluide, les différentes explications de l'énurésie, les maladies urinaires, les couleurs, substances et dépôts de l'urine, en empruntant à Hippocrate, à Galien, mais aussi à des traités plus tardifs d'origine byzantine ${ }^{12}$, eut, après avoir été traduit en latin par Constantin l'Africain dans la seconde moitié

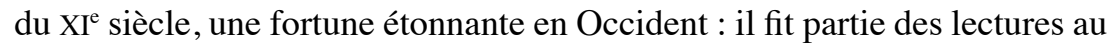
programme pour les étudiants de médecine des principales universités, et les maîtres en rédigèrent des commentaires. Ce succès s'explique par le fait que ni Galien ni aucun auteur arabe traduit en latin n'avait écrit avant lui de traité spécifique sur le sujet.

L'importance de l'examen des urines dans la médecine arabe peut se mesurer à la précision du protocole à respecter : diverses règles étaient dictées afin que la significatio de cet examen se réalise pleinement, pour parler comme Avicenne traduit par Gérard de Crémone (m. 1187) au $\mathrm{XII}^{\mathrm{e}}$ siècle $^{13}$. Pour ceux qui scrutaient l'urine en effet, les humeurs, tant qu'elles étaient dans le corps à l'état naturel, n'avaient pas de signification en soi, faute d'avoir une existence sensible : c'est par leurs transformations à l'intérieur du corps dont elles revêtaient les qualités qu'elles se chargeaient d'une signification, susceptible d'une interprétation lors de leur sortie hors du corps, qui les soumettait aux sens de l'observateur ${ }^{14}$.

9. Sur ce personnage fondamental et ses traductions de Galien en syriaque ou en arabe (il accéda à cent vingt-neuf textes de Galien), on verra par exemple D. JACQUART et F. MicheAu, La Médecine arabe et l'Occident médiéval..., p. 36 sq., et G. TROUPEAU, « Les débuts de la médecine arabe : les grandes familles de médecins. Hunayn, la formation du vocabulaire », dans La Médecine au temps des califes : à l'ombre d'Avicenne, Catalogue de l'exposition, Paris, 1996, p. 41-46.

10. D. JACQUART, « La place d'Isaac Israeli dans la médecine médiévale », Vesalius (special number, 1998), p. 19-27 (p. 19 et 22).

11. Son Traité des fièvres est ainsi la première monographie arabe sur la question, traduite par la suite en latin, en hébreu et en castillan ; $f$. D. JACQUART et F. MICHEAU, La Médecine arabe et l'Occident médiéval..., p. 111.

12. Ibid., p. 111.

13. A viCENnE, Canon medicinae, Venise, 2 vol., 1527, Summa II, doctr. III, fen 2, cap. 1, t. I, p. 150 : « donec hypostasis separetur et significatio compleatur ».

14. On paraphrase ici les propos du médecin catalan Arnaud de Villeneuve (m. 1311) : « Humores autem dum in corpore existunt naturaliter non possunt per se significare, propterea 
Un récipient approprié était utilisé pour l'examen : tous les auteurs recommandent un verre mince et blanc parfaitement translucide, ainsi qu'un flacon propre, lisse, dépourvu de toute saillie susceptible d'altérer la transparence. Et il faut bien distinguer ici l'urinal, la matula servant à l'examen, du pot de chambre ou vase de nuit qui, lui, n'avait pas besoin d'être transparent. La matière de l'urinal se devait d'être non colorée, afin de ne pas altérer la teinte de l'urine ; en d'autres termes, il devait être soit en cristal, soit en verre blanc, mais en aucun cas d'une matière grossière, comme le verre vert ou le verre blanc épais, à travers lequel le regard ne pouvait pénétrer avec la même subtilité.

Alors qu'il se présente comme un récipient cylindrique dans certaines miniatures d'origine byzantine ${ }^{15}$, le flacon change de forme dans le monde arabo-musulman et occidental ; il est désormais pansu et doté d'un col qui se développa. La forme du vase était censée être celle de la vessie ${ }^{16}$, rond à la base, de telle sorte qu'on pensait observer l'urine dans la position où elle se présentait à l'intérieur du corps. La forme pouvait en effet influer sur le jugement : ainsi dans un flacon trop large, si l'on avait peu d'urine, on ne pouvait rien dire de la situation des dépôts dans le liquide et, inversement, un flacon trop étroit empêchait d'avoir la matière à suffisance. Pour l'inspection approfondie des sédiments, al-Rāzī conseillait ainsi de « recueillir l'urine dans un grand flacon blanc, dont la base est ronde, et de l'y laisser pendant trois à dix heures pour que s'y déposent les sédiments » (Aph. 198) ${ }^{17}$. Outre sa matière, sa transparence et sa propreté, la taille de l'objet requérait donc toute l'attention, afin d'éviter toute déperdition d'urine.

On recueillait le fluide de préférence le matin : pendant le sommeil, comme le dit Isaac $^{18}$, se concentre ce qui se disperse ensuite à l'état de

quia sensui non subjiciuntur, sed per aliud scilicet per immutationem quam faciunt in qualitate corporis - scilicet colore, odore, sapore, duricie, mollicie, et ceteris ; et ideo quantum ad hoc eorum significationes continentur in significationibus sumptis ex qualitatibus corporis. Secundum autem quod humores egrediuntur a corpore tunc necessario habent significare ut exeuncia a corpore et ideo quantum ad hoc significatio reservabitur usque ad significationem ab exeuntibus sumptam » (ARNAUD DE VILLENEUVE, Tractatus de considerationibus operis medicine sive de flebotomia, éd. L. Demaitre, intro. P. GIL-Sotres, Barcelone, 1988, p. 169).

15. Voir par exemple la miniature du ms. Bologna, Biblioteca Universitaria, 3632, f. 51, représentant Théophile recevant un récipient cylindrique des mains de son assistant Posos, et qui sert d'illustration de couverture à l'ouvrage $I l$ De urinis di Teofilo Protospatario... cité n. 5. Même Isaac Judaeus est représenté avec un urinal de ce type dans ce même ms., f. 134 v.

16. «Ad vesicae formam », dit le texte latin d'Isaac; $c f$. ISAAC, Liber urinarum, II ${ }^{\mathrm{a}}$ particula, éd. J. PEINE, Die Harnschrift des Isaac Judeus, Leipzig, 1919, p. 15, 1. 224.

17. Cité par D. JACQUART et G. TROUPEAU, « La consultation médicale : de l'observation du malade à la prescription », dans La Médecine au temps des califes..., p. 77-81 (p. 78).

18. Cf. ISAAC, Liber urinarum, $\mathrm{II}^{\mathrm{a}}$ particula, p. 14, 1. 177 : « urina noctis apertiorem habet significationem quam diei ; quia, dum quis vigilat, natura exit ad corporis exteriora, ubi se dividit ad quinque sensuum explenda officia ». 
veille sous l'effet des cinq sens, et c'est pourquoi l'urine du matin passait pour la meilleure. Pour l'inspecter, selon al-Rāzī, il fallait « la prendre après que le malade s'est réveillé d'un long sommeil et avant qu'il ne boive quelque chose, sinon l'état de l'urine se détériore » (Aph. 196) ${ }^{19}$. Un temps de repos, de décantation était nécessaire : Avicenne estimait qu'il fallait l'examiner dans l'heure, sous peine de modification du sédiment, tandis qu'Isaac préconisait de l'examiner à trois reprises, à une heure d'intervalle.

La lumière du soleil était fondamentale ${ }^{20}$, mais ses rayons ne devaient pas toucher l'urine, pas plus d'ailleurs que le vent, susceptible de la refroidir, précise Avicenne ${ }^{21}$, qui explique également comment l'épaisseur ou la clarté du liquide augmente aux yeux de celui qui l'examine selon qu'il éloigne ou rapproche de lui le flacon. Vent ou chaleur étaient autant de facteurs susceptibles d'altérer son contenu.

Le médecin observait la couleur du liquide, sa quantité, son degré de clarté, ses sédiments, le volume qu'ils occupaient et la manière dont ils se comportaient. «Quand la maturation est complète, les sédiments se déposent en bas du flacon ; quand elle est moyenne, ils sont suspendus, et quand elle est à ses débuts, ils surnagent », indiquait al-Rāzī (Aph. 201). Quant à la couleur et la consistance de l'urine, elles étaient censées renseigner sur l'état de coction du sang, puisqu'on expliquait l'origine de l'urine en invoquant l'existence de trois digestions consécutives dans le corps, et donc de trois superfluités : la digestion opérée dans l'estomac donnait les excréments, l'urine pour sa part était censée provenir de la seconde digestion, celle qui s'opérait dans le foie, et la troisième digestion, dans les tissus, produisait théoriquement peu de superfluités, sauf chez les malades. Chez ces derniers, en s'expulsant avec l'urine, les superfluités formaient l'hypostase, ou suspension, et d'une manière générale les contenta dont le praticien tirait un enseignement quant à l'état du corps. Comme le dit encore al-Rāzi : «Si la coction est insuffisante, l'urine est blanche et fine ; si elle est excessive, l'urine est rouge et épaisse ; si elle est moyenne, l'urine est jaune, d'épaisseur et de finesse moyennes ; si la chaleur est excessive dans le foie, l'urine est noire et très épaisse, comme c'est le cas dans les maladies aiguës mortelles » (Aph. 194) ${ }^{22}$.

Le médecin appréciait également la viscosité de l'urine et sa consistance, sans oublier son odeur, voire son goût - Rhazès avait souligné l'importance du toucher et du goût de l'urine, et Avicenne, celle de l'odeur :

19. Cité par D. JACQUART et G. TROUPEAU, « La consultation médicale... », p. 77.

20. ISAAC, Liber urinarum, p. 15,1.233 : «oportet ergo, ut, cum a vesica exierit, videatur, antequam ab aere corrumpatur, in loco lucido splendori solis opposito ».

21. Avicenne, Canon medicinae, Summa II, doctr. III, fen 2, cap. 1, p. 150 : « ne sol eam tangat, neque ventus, ne perturbetur, et ne congeletur » $[. .$.$] « oportet ut in lumine aspiciatur,$ ita quod super eam non cadat radius ».

22. Cité par D. JaCQUART et G. TROUPEAU, « La consultation médicale... », p. 77. 
selon lui, une urine inodore était signe de complexion froide et de crudité superflue $^{23}$. Tous ces traits étaient rapportés aux symptômes de différentes maladies et la couleur, en particulier, permettait un diagnostic sur les conditions des humeurs et de la digestion.

\section{Les limites de l'examen d'un fluide}

L'inspection de l'urine était considérée comme un élément essentiel de l'examen clinique, qui devait permettre au médecin d'établir son diagnostic, mais qui n'éclipsait pas pour autant l'interrogation du malade, la palpation du pouls, ou l'observation des signes et des symptômes ${ }^{24}$. Certains émirent de fait très tôt des réticences quant au risque d'illusion d'un examen clinique réduit à la seule analyse des urines. Ainsi dès le $\mathrm{IX}^{\mathrm{e}}$ siècle, Yūhannā Ibn Māsawayh (v. 777-857), un médecin originaire de Gundeshāpūr qui exerça à Badgad et eut Hunayn pour disciple, exprime certaines réserves dans ses aphorismes (ou axiomes médicaux). Il conseille au sage « qu'il abandonne la suffisance des médecins et leur délire devant les flacons et qu'il n'ait pas honte d'interroger les malades » (Aph. 43), car selon lui « l'urine est le signe le plus trompeur, à moins que la maladie ne siège à l'intérieur des veines » (Aph. 42). Il estime que « lorsque le médecin n'a pas inspecté l'urine du malade dans son état de santé, il n'approche pas de la vérité dans son traitement »(Aph. 112) et il constate que « les urines qui indiquent la santé ne sont pas, chez tous les gens, uniques en couleur et en consistance » (Aph. 111) ${ }^{25}$. Yūhannā relativisait donc l'efficacité de l'urine pour déterminer l'état de santé du patient, et soulignait que le moyen le plus sûr était d'interroger longuement celui-ci, et de l'examiner.

Des anecdotes célèbres disent de fait à la fois le rôle de l'analyse des urines dans la médecine arabe, mais aussi ses risques, comme le destin d'Abū Qūraysh 'İsā ibn Yahyāa, un apothicaire du VIII ${ }^{\mathrm{e}}$ siècle élevé par le calife au rang de médecin pour avoir su lire dans une fiole que sa favorite était enceinte d'un mâle appelé à régner ${ }^{26}$. Il tenait boutique près du palais et un jour de l'année 760 une servante lui apporta un flacon à examiner, afin qu'il diagnostique la maladie de sa maîtresse. Il prédit alors : « Cette

23. Voir Canon medicinae, Summa II, doctr. III, fen 2, cap. 4, t. I, p. 156 : « et nos quidem dicemus, quod cum urina nullo modo odorem habet, complexionis frigiditatem significat, et superfluam cruditatem ».

24. D. JaCQUART et G. Troupeau, « La consultation médicale... », p. 77.

25. Tous ces aphorismes sont cités par D. JACQUART et G. TROUPEAU, « La consultation médicale... », p. 78. On se reportera à Yūhannā Ibn Māsawayh [Jean Mesué], Livre des axiomes médicaux (Aphorismi), édition du texte arabe et des versions latines avec traduction française et lexique, par D. JACQUART et G. TROUPEAU, Genève, 1980.

26. Cité par M. Neuburger, « The Early History of Urology », Bulletin of the Medical Library Association, 25-3 (1937), p. 147-165 (p. 156). 
dame est enceinte. Elle aura un enfant mâle. Il est appelé aux plus hautes destinées et règnera un jour. » Or la prédiction se réalisa. La maîtresse était la favorite du futur calife al-Mahdī, et l'enfant attendu deviendra lui-même calife sous le nom d'al-Hādī. Du coup, le pharmacien fut promu médecin officiel de la princesse, malgré son incompétence.

Une situation ayant un ressort comparable est rapportée par Raymond Le $\mathrm{Coz}^{27}$ : toujours à la fin du VIII ${ }^{\mathrm{e}}$ siècle, quand le calife Harūn al-Rashīd tomba malade, on fit appel au syriaque Bakhtīshū' ibn Djūrdjis (Gurgis), qui exerçait à Gundeshāpūr. Comme l'ont souligné Danielle Jacquart et Françoise Micheau, les califes, de fait, «n'avaient pas attendu la traduction des grandes œuvres de l'Antiquité pour chercher auprès des médecins formés à bonne école des remèdes à leurs maux », et « l'influence de la médecine grecque à la cour des premiers Abbassides apparut d'abord grâce à des hommes venus de Gundisabur ${ }^{28} \gg$. Souhaitant tester les connaissances du nouvel arrivé, Harūn al-Rashīd demanda à trois médecins de lui faire passer un examen. L'un d'eux, jaloux mais lucide, lui aurait fait savoir qu'aucun d'eux n'en était capable et que Bakhtīshū' leur était supérieur. Le calife se chargea alors lui-même du «test » et demanda au nouveau venu d'examiner l'urine de sa favorite en vue de lui prescrire une médecine. Le calife lui avait présenté l'urine d'une bête de somme, mais le candidat éventa sa ruse, et prescrivit une mesure d'orge. Ravi de sa réponse, et rassuré sur ses compétences, le calife le nomma médecin en chef du palais.

Une mise à l'épreuve du praticien par le patient était donc possible, et un thème comme celui de la falsification de l'urine par l'ingestion de safran ou d'herbes susceptibles de la colorer est récurrent dans les écrits des médecins : Rhazès et Avicenne mettent ainsi en garde contre aliments et boissons pouvant altérer (délibérément ou non) la couleur du fluide tels la betterave ou le safran, voire contre l'usage du henné, susceptible lui aussi de modifier sa couleur ${ }^{29}$. Quant aux développements sur les urines animales, ils avaient eux aussi pour but d'éviter au praticien de se laisser prendre $^{30}$. Leur connaissance s'avère d'autant plus utile que nombre d'entre

27. Voir R. LE Coz, Les Médecins nestoriens au Moyen Âge. Les maîtres des Arabes, Paris, 2004, p. 106.

28. D. JACQUART et F. MicheAU, La Médecine arabe et l'Occident médiéval..., p. 35.

29. A viCENNE, Canon medicinae, Summa II, doctrinae III, fen 2, cap. 1, t. I, p. 149-150 : « et non sit, qui in cibo, aut potu rem tingentem accepit, sicut crocum, et cassiam fistulam ; quoniam urinam tingendo ad citrinitatem et rubedinem mutant, et sicut olera que tingendo mutant ad viriditatem », etc. Signalons que, dans les années 930, le médecin égyptien Nastas ben Jurayh, dans une Épitre sur l'urine en arabe au médecin chrétien Yazīd ben Rūmān, expliquait comment reconnaître les urines d'un juif... le samedi [traduction de cette lettre dans J. VERNET, « Los médicos andaluces en el Libro de las generaciones de médicos de Ibn Gulgul », Anuario de Estudios medievales, 5 (1968), p. 445-462 (p. 455)].

30. Avicenne, Canon medicinae, lib. I, fen 2, doctr. 3, cap. 11, t. I, p. 160 : « plerumque confert medico, quum animalium urinas cognoscit ; propterea quod, quum ipse in eis 
elles servent de points de comparaison pour décrire celles des humains, telle l'urine jumenteuse, subjugalis, et Avicenne y consacre de nombreuses pages, de même qu'au distinguo entre urines féminines et masculines.

On l'a dit, l'uroscopie devint accessible aux médecins latins par un double canal, la traduction en latin de Théophile d'une part et celle d'Isaac d'autre part au $\mathrm{XI}^{\mathrm{e}}$ siècle. La médecine occidentale tendit à demander à l'examen du liquide de plus en plus d'informations, non plus seulement sur les fièvres, le foie ou les voies urinaires, mais sur l'état de santé du corps tout entier. L'examen des urines du patient en vint à jouer un rôle central dans la consultation médicale et la lecture du fluide recueilli se mua en acte interprétatif par excellence de la médecine d'alors, la palpation du pouls étant jugée trop difficile par beaucoup de praticiens, et l'interrogatoire du malade pas toujours réalisé, puisque la consultation pouvait se faire à distance, par lettres ou par examen d'un urinal.

De fait, une des particularités, riche de conséquences, de la consultation uroscopique telle qu'elle se développa en Occident, résidait, on l'a dit, dans la possibilité pour le patient de ne pas y être physiquement présent : il était souvent plus facile d'acheminer un flacon qu'un malade, et s'il n'était pas en état de se déplacer, ce dernier n'était représenté que par son urinal confié à un messager qui pouvait être un serviteur, un parent ou un ami. Le porteur (qui pouvait aussi être une femme) amenait l'urinal dans un petit panier doté d'un couvercle et d'une anse ${ }^{31}$, et c'est lui qui fournissait des précisions sur l'état du malade, une fois que le médecin avait donné les premières conclusions de son diagnostic au vu de l'urine.

Avec l'élimination d'une partie importante de la consultation - l'examen clinique et l'interrogatoire du malade et de son entourage - et l'introduction d'un double système de représentation du patient - l'urinal métonymique du malade et le messager porte-parole faisaient désormais face au praticien -, la consultation médicale put se muer durablement en jeu de rôles. Vers 1400, le médecin parisien Guillaume Boucher ne fut-il pas lui aussi ridiculisé pour s'être trompé sur le sexe de son patient, comme le révèle un de ses Consilia ? «Il dit, quand il vit l'urine, qui était assez blanche, que [le patient] avait l'estomac faible, qu'il ne pouvait digérer, et qu'il s'agissait d'un homme. Les porteurs se mirent à rire et dirent que

experitur, certitudinem facit, et hoc quidem difficile est. Quidam autem dixerunt, quod urina asini est sicut butyrum liquidum cum turbiditate, et grossitie exteriore. Et subjugalium urinae ei similantur : sed sunt citrinae, et videbitur, quod superior medietas urinalis sit clara, et inferior medietas sit ».

31. Une iconographie riche nous en a conservé le souvenir, mais aucun témoin n'a survécu, selon R. C. Tyson, Medieval Glass Vessels in England AD 1200-1500 : a Survey, Doctoral thesis, Durham University, 1996, p. 7. 
c'était une femme ${ }^{32}$. » L'urine blanche pouvait certes signifier différentes choses, notamment un problème à l'estomac...

À en croire les Cautèles des urines anonymes, la substitution d'urines de femme à celles d'un homme restait le premier moyen d'éprouver la validité du médecin, qui devait songer à s'en prémunir, et la littérature exploita ce thème, comme avec le Décaméron de Boccace ${ }^{33}$. Mais la substitution d'urines de femme à celle d'un homme n'était qu'une des nombreuses possibilités de tromperie qu'offrait cet examen, bien que la plus emblématique.

$\mathrm{Au} \mathrm{XIV}$ siècle, le prédicateur dominicain Philippe de Ferrare met en scène un patient ayant voulu tromper son médecin en remplissant son urinal de vin blanc ${ }^{34} . .$. , et au siècle suivant, le Padouan Michel Savonarole, conscient des possibilités de tromperie qu'offrait le seul critère de la couleur, surtout en cas d'urine verte ou livide, éminemment bivalentes, recommandait au praticien de compléter son examen, notamment par la prise en compte du pouls du patient et de l'odeur de l'urine ${ }^{35}$. Les pièges de l'urine sont en effet encore une réalité au XV siècle, si l'on en croit aussi les conseils du maître parisien Jacques Despars aux médecins débutants : «En outre, je conseille aux jeunes médecins d'éviter le plus possible l'utilisation fréquente de purgatifs, de prononcer tardivement et avec précaution leur pronostic en cas de danger, et de dire peu à propos de l'inspection des urines. En effet, on est souvent trompé par celle-ci, diffamé, et on se voit parfois attribuer toute sa vie maigres réputation et respect ${ }^{36}$. » En matière de jeux de rôles et de duperie autour de l'inspection des urines, la médecine occidentale hérita donc de failles déjà mises en relief par certains auteurs arabes dès le $\mathrm{IX}^{\mathrm{e}}$ siècle, et les creusa encore avec l'essor de la consultation in absentia patientis.

32. $C f$. E. WICKERSHEIMER, «Les secrets et les conseils de maître Guillaume Boucher et de ses confrères. Contribution à l'histoire de la médecine à Paris vers 1400 », Bulletin de la Société française d'Histoire de la médecine, 8 (1909), p. 199-305, n 61, p. 285-289 ; voir à ce sujet D. JACQUART, La Médecine médiévale dans le cadre parisien, Paris, 1998, p. 502.

33. Voir par exemple BoCCACE, Décaméron, Neuvième journée, Troisième nouvelle, trad. V. BRANCA, Paris, 1953, p. 769-770.

34. Sur cet auteur, on verra le récent article de J. ZIEGLER, « Medicine and the Body at the Table in Fourteenth-Century Italy : Book one of Philip of Ferrara's Liber de introductione loquendi », dans F. GLAZE, B. NANCE éd., Between Text and Patient : The Medical Enterprise in Medieval and Early Modern Europe, Florence, 2011, p. 121-136.

35. Cf. Michaelis Savonarolis de urinis summa, dans Practica canonica de febribus Io. Michaelis Savonarole, eiusdem de pulsibus, urinis, egestionibus, vermibus, balneis, Venise, 1552, f. 114ra.

36. Cité par D. JACQUART, La Médecine médiévale dans le cadre parisien..., p. 441. 


\section{L'uroscopie en images}

On peut peut-être, pour achever cet aperçu sur les différences et les points communs entre Orient et Occident à propos de l'examen des urines, se tourner vers l'iconographie et soulever la question des fortunes de ce thème dans les aires concernées. Je n'ai en effet à ce jour connaissance d'aucune illustration de l'examen des urines dans un manuscrit arabe, ce qui ne veut pas dire qu'il n'en existe pas. Par exemple, dans un manuscrit de la Burgerbibliothek de Berne réalisé en Italie entre 1195 et 1197, qui transmet le Liber ad honorem Augusti sive de rebus Siculis de Pierre d'Eboli (11601220), figure le portrait d'un praticien arabe. La miniature du folio 97 recto présente la maladie et la mort du roi normand Guillaume II (18 novembre 1189). Le souverain est représenté d'abord malade, Rex Wilhelmus egrotans, sous les deuxième et troisième arcades, sur un lit. Un médecin du nom d'Achim (Achim medicus), assis à la gauche du roi, observe un vase avec ses urines, et la présence d'un médecin arabe atteste l'importance que la cour normande accordait à la médecine du monde islamique et à sa science en général ; à la gauche du roi, un astrologue, astrologus, lui aussi arabe comme le suggèrent le turban et le menton barbu, tient dans sa main gauche un astrolabe et, dans la droite, un livre, peut-être des tables astronomiques ${ }^{37}$.

Une autre figure de médecin supposément arabe se trouve dans un manuscrit en hébreu du Canon d'Avicenne, copié à Bologne vers 1320$1330^{38}$ : illustrant la phrase initiale du $4^{\mathrm{e}}$ livre sur les fièvres, une vignette représente un médecin qui prend le pouls de son patient, ce dernier tenant en main un flacon d'urine pour un examen ultérieur prescrit par Avicenne dans la suite du texte. Quant au manuscrit latin 6919 de la BnF, un exemplaire du Canon medicinae copié en Italie au XIII ${ }^{\mathrm{e}}$ siècle, il offre aussi un portrait du savant avec un urinal au folio 212, de même que le Canon transmis par le manuscrit Gaddi 24, qui contient une scène d'uroscopie au folio 125 recto, ou le manuscrit $n^{\circ} 9$ de la Hunter Library de Glasgow, folio 84, où l'on voit une femme, peut-être enceinte, tendre son flacon à un médecin. On mentionnera aussi quelques représentations de Constantin l'Africain mirant les urines, comme dans le manuscrit Oxford, Bodleian Library, Rawl C 328, folio 3 , où il est entouré de femmes venues lui soumettre la leur et espérant peut-être un diagnostic de grossesse, ou au premier folio du manuscrit Paris, BnF, lat. 6884, qui renferme la traduction latine du De urinis d'Isaac. Constantin n'était pas à proprement parler l'auteur d'un traité d'uroscopie, mais son nom était attaché à la traduction du De urinis d'Isaac, et même la

37. Bern, Burgerbibliothek, ms. 120 II : miniature reproduite entre autres dans G. D’AndirAn éd., La Médecine ancienne, du corps aux étoiles, Cologny/Paris, 2010, p. 272.

38. Paris, BnF, ms. hébreu 1146 ; image reproduite dans La Médecine au temps des califes..., p. 304. 
légende le concernant, racontée par un « Mathieu F. (Ferrarius ?), lui prête un certain savoir en la matière : d'après lui, Constantin était un marchand musulman venu une première fois à Salerne au temps du dernier prince lombard Gisulf II (1052-1077) ; il y aurait manifesté quelque compétence dans l'analyse des urines ${ }^{39}$. Le manuscrit Univ. Library, 170 (D.b.VI.1) d'Édimbourg, un exemplaire du Breviarium de Serapion, contient une scène d'uroscopie, au folio 87 verso, de même que le manuscrit Supplément persan 2051 de la BnF, qui renferme l'œuvre d'un juriste et homme de lettres arabe, al-Qazwīnī, mort en 1283 ('adjā'ib al-makhlūqāt, alias Les Merveilles des choses créées et les curiosités des choses existantes), et qui campe également un médecin en train d'examiner des urines au folio 158 verso (fig. 1). Mais il s'agit ici non d'une œuvre arabe mais d'une traduction persane de l'œuvre.

La moisson, à ce jour, est maigre, voire nulle en ce qui concerne les manuscrits arabes, ce qui semble confirmer ce qu'on sait par ailleurs de l'illustration médicale dans le monde arabe : loin d'être absente, elle ne figure que dans certains types d'ouvrages : dictionnaires de remèdes, traités sur la chirurgie, plus rarement anatomie ${ }^{40}$. En Occident, au contraire, la scène de consultation uroscopique fut si abondamment représentée à partir du XII ${ }^{\mathrm{e}}$ siècle qu'elle a fait l'objet d'un livre entier de Friedrich von Zglinicki ${ }^{41}$. L'urinal et son contenu furent jugés dignes d'une représentation esthétique et le célèbre récipient accompagna des textes très différents, avec deux grands types d'emploi : d'une part, la représentation d'une grande figure, historique, littéraire ou mythique, en particulier de grands savants, d'autre part, la scène typique de consultation uroscopique mettant en scène un médecin et un ou plusieurs malades. Les représentations d'urinal ne sont pas absentes des traités de médecine, où elles figurent souvent dans une lettrine, et bien sûr dans les « roues des urines », représentant les différentes couleurs du fluide comme autant de flacons disposés en roue, du blanc au noir - un schéma dont je ne connais pas d'équivalent pour le monde arabe (fig. 2). Mais des ouvrages littéraires accueillent aussi des images d'urinal, et notamment des écrits historiques ; des représentations d'urinal ornent également des livres d'heures, ou apparaissent dans l'encadrement. Dans ce dernier cas, le dessin n'est pas toujours dénué d'humour, comme dans les marginalia de différents manuscrits : l'urinal a acquis le statut d'attribut et est au médecin en quelque sorte ce qu'est la palme au martyre.

39. Rapporté par D. JACQUART et F. MiChEAU, La Médecine arabe et l'Occident médiéval..., p. 98.

40. Cf. M.-G. BALtY-GueSDON, « Le livre médical et son illustration », dans $L a$ Médecine au temps des califes..., p. 231-238 (p. 231).

41. F. VON ZGLINICKI, Die Uroskopie in der bildenden Kunst. Eine kunst- und medizinhistorische Untersuchung über die Harnschau, Darmstadt, 1982. 


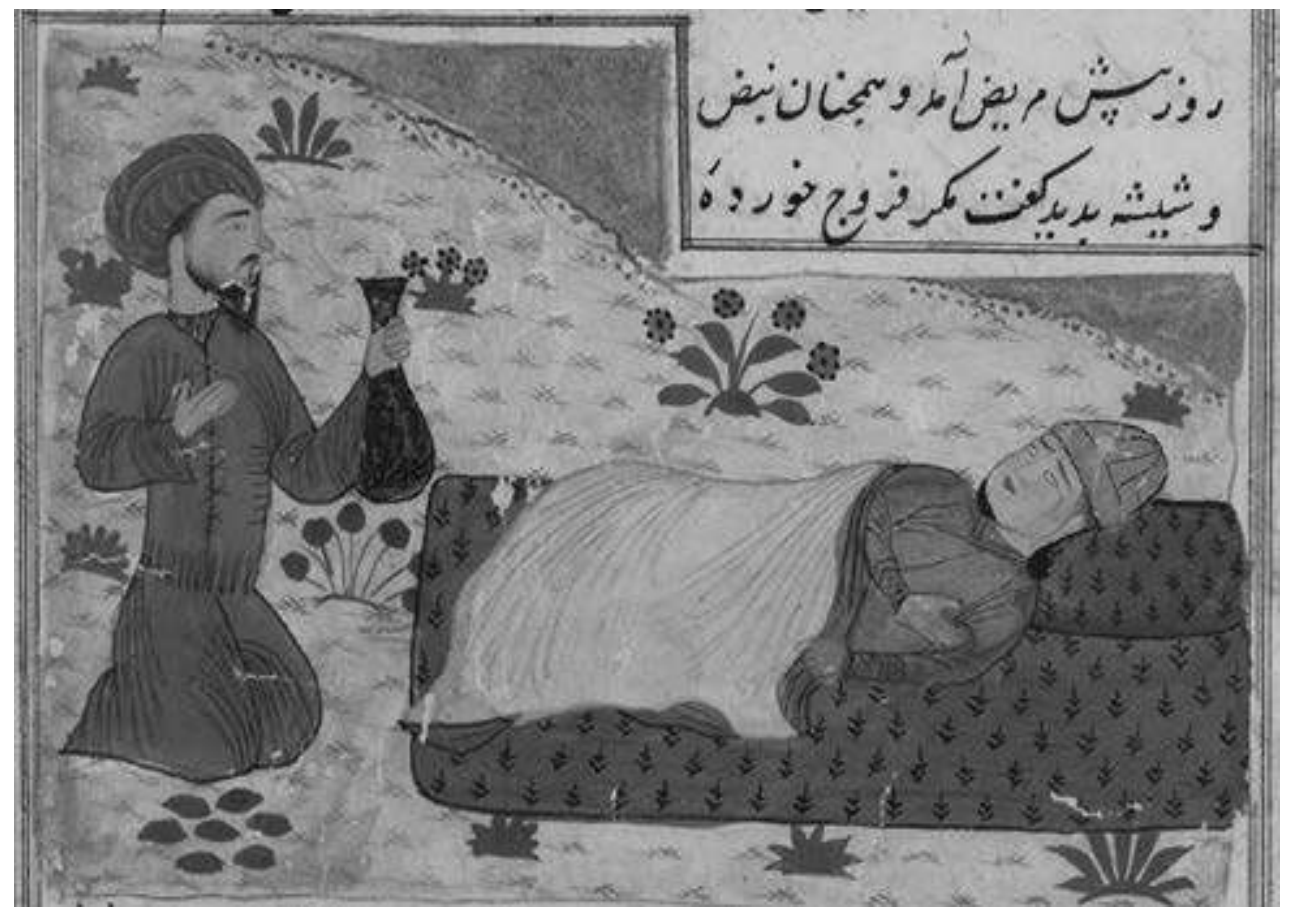

Fig. 1. Médecin examinant des urines

(C) Paris, BnF, ms. Supplément persan 2051, folio 158v.)

Les miniatures accueillirent ce motif sans discontinuer jusqu'à la fin du Moyen Âge, et furent ensuite relayées par les gravures accompagnant les premiers ouvrages sortis des presses. Mais les images d'urinal qui nous sont parvenues du monde occidental ne sont pas cantonnées entre les pages des livres, et le thème gagna différents arts plastiques ou supports. Dès le XIII ${ }^{\mathrm{e}}$ siècle, dans la crypte de la cathédrale d'Anagni, par exemple, le fameux récipient agrémente la représentation d'Hippocrate délivrant son enseignement à Galien, et à Sienne il figure dans une fresque de l'hôpital Santa Maria della Scala peinte vers 1443 . Il peut également se donner à voir sur des façades d'édifices : sur la face sud du campanile du Dôme de Florence, un bas-relief d'Andrea Pisano représente la médecine incarnée par un praticien tenant une matula à contre-jour ; à Pistoia, un bas-relief en terre cuite émaillée réalisé par les della Robbia sur la façade de l'hôpital del Ceppo, dans la première moitié $\mathrm{du}_{\mathrm{XV}}^{\mathrm{e}}$ siècle, représente un urinal. Enfin, le médecin tenant sa matula est aussi parfois représenté sur les miséricordes des stalles d'église, souvent de manière satirique quand il revêt la forme d'un singe, symbole de vanité et de sottise... N'allongeons 


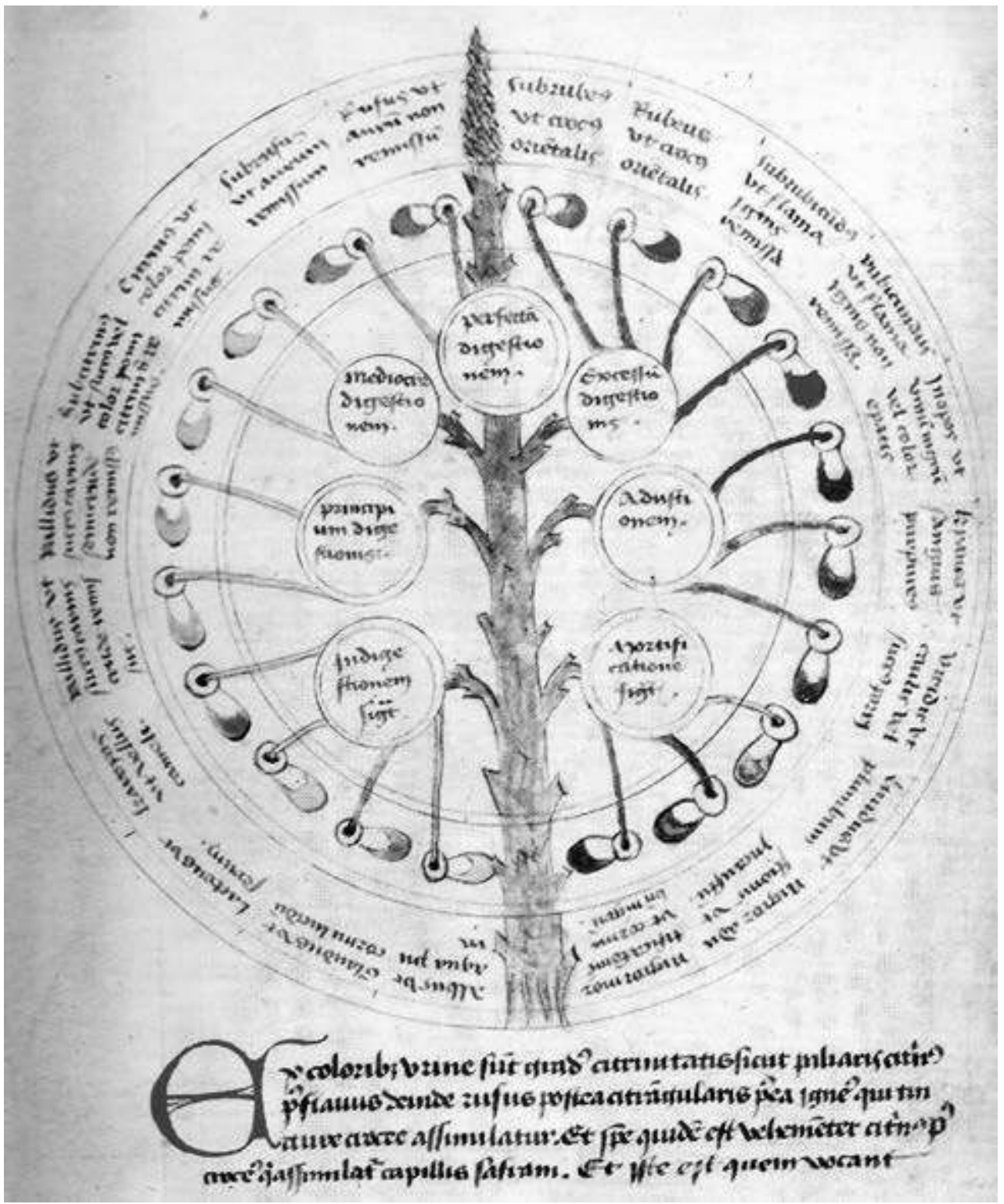

Fig. 2. Roue des urines, Recueil de traités de médecine en latin et en français,

(début du XV siècle)

(C) Paris, BnF, ms. lat. 11229, folio 19v.) 
pas l'énumération ${ }^{42}$. Retenons qu'en Occident, l'examen des urines fut représenté sur des supports divers, alors que d'autres gestes médicaux aussi courants que le toucher du pouls, et surtout la saignée, inspirèrent nettement moins les artistes.

Au Moyen Âge, l'urine relève incontestablement $d u$ « bas corporel », en Occident comme dans l'espace arabo-islamique. Dans cette dernière aire culturelle, par exemple, l'émission d'urine était un cas d'impureté rituelle obligeant à renouveler ses ablutions avant l'office ${ }^{43}$. Or aucune matière vile du corps n'a suscité tant d'expressions figurées, voire artistiques, en Occident. En Orient en revanche, où la science des urines avait pris corps plus tôt, on rencontre peu de représentations de scène de consultation et surtout nulle trace des fameuses roues des urines. Est-ce le signe d'une défiance précoce des médecins arabes vis-à-vis de l'observation de l'urine, si l'on se souvient que, dès le IX ${ }^{\mathrm{e}}$ siècle, Ibn Māsawayh avait mis en garde contre la confiance excessive accordée aux informations tirées de cet examen ? Ou cette rareté, voire cette absence, ne peut-elle finalement pas se rapporter à la place différente accordée à l'illustration dans la littérature médicale arabe par rapport à celle d'Occident ? Car si d'autres gestes, comme la saignée ou la prise de pouls, ont fait l'objet de miniatures dans des manuscrits arabes ${ }^{44}$, de telles scènes s'avèrent rarissimes dans les traités de médecine ${ }^{45}$. L'examen des urines, de ce point de vue, et dans la limite de mon information, ne semble pas avoir eu dans le monde musulman un traitement différent de celui d'autres gestes médicaux plus ou moins quotidiens, alors que l'Occident médiéval se caractérise par une surreprésentation de la scène de consultation uroscopique sur différents supports jusqu'à la fin de

42. Pour en savoir plus, on se reportera à l'étude de Friedrich von Zglinicki cité à la note précédente. On me permettra de renvoyer aussi à L. MOULINIER-BROGI, L'Uroscopie au Moyen Âge. «Lire dans un verre la nature de l'homme », Paris, 2012, $\mathrm{II}^{\mathrm{e}}$ partie.

43. Voir J. ReVAult, "Deux mīd'ās tunisoises (XV et XVII ${ }^{\mathrm{e}}$ siècles) », Revue de l'Occident musulman et de la Méditerranée, 15-16 (1973), p. 275-290. L'émission d'urine, comme celle de gaz ou encore les vomissements, était une impureté rituelle rendant nécessaire une nouvelle ablution.

44. Certaines sont reproduites dans le catalogue de l'exposition La Médecine au temps des califes... : une scène de prise de pouls dans un ms. du Kalila et Dimna, réalisé à Bagdad en 1343, p. 48 ; médecin prenant le pouls d'une patiente dans un ms. de l'école de Shiraz, 1410, p. 278 ; ou deux scènes de saignée illustrant les Maqāmāt, des contes plaisants d'al-Harīrī (m. en 1122), réalisées respectivement en Iraq, 1240, et à Bagdad 1237 (voir p. 90-91).

45. Il n'y a guère que dans un manuscrit aujourd'hui à Ham dans le Surrey (Keir collection), exécuté à Bagdad en 1244, un feuillet provenant d'une traduction arabe de Dioscoride que l'on trouve, à côté des représentations traditionnelles de plantes médicinales, des scènes où figurent des personnages, en particulier un interrogatoire de patient et une saignée ; mais d'examen des urines, point de trace ici : voir la notice dans La Médecine au temps des califes..., p. 254. 
la période. L'impureté représentée par l'urine en Islam peut jouer un rôle dans ce contraste, et l'on n'exclura donc pas une influence religieuse. Mais quoi qu'il en soit, si les quelques représentations de médecins arabes tenant un urinal proviennent du monde occidental, n'est-ce pas aussi un indice de l'image de spécialistes de l'uroscopie, d'auctoritates en la matière, que les Latins se faisaient d'eux?

Laurence Moulinier-Brogi - Université Lumière-Lyon 2/UMR 5648 - CIHAM

\section{L'examen des urines dans la médecine médiévale en terre d'Islam et en Occident. Un aperçu}

Cet article a pour objet non pas tant des lieux d'aisance que le produit d'une évacuation corporelle, à savoir l'urine, et son importance dans la médecine du Moyen Âge en terre d'Islam et en Occident. Le diagnostic comme le pronostic médical se fondaient en effet sur l'inspection de ce fluide recueilli dans un flacon particulier, l'urinal. Mais l'examen des urines n'avait pas toujours eu une telle importance dans la sémiologie : la part croissante de l'uroscopie dans l'examen clinique est liée à l'œuvre du Byzantin Théophile, dont le Peri ouron influença dans un premier temps la médecine byzantine et la médecine arabe : dès le $X^{e}$ siècle, le médecin Isaac Israëli (Ishāq al-Isrā'îī) consacra aux urines la première monographie en arabe sur le sujet. Puis, après que les traités de Théophile et d'Isaac eurent été traduits en latin au $\mathrm{XI}^{\mathrm{e}}$ siècle, la médecine occidentale fit sien leur enseignement en la matière. Après avoir rappelé les fondements de l'uroscopie dans la médecine occidentale et dans celle dite arabe, et en se fondant sur les apports textuels, mais aussi iconographiques, on tente ici de comparer l'importance de l'inspection des urines dans ces deux espaces, et les brèches que les modalités d'un tel examen pouvaient ouvrir dans la relation entre malades et praticiens.

Diagnostic - examen clinique - Isaac - Israeli - médecine - médecine arabe - pronostic - sémiologie médicale -Théophile - protospathaire - urines urinal - uroscopie

\section{The Urine's Examination in Medieval Medicine, between Islamic and Occidental Worlds. A Short Overview}

This paper does not focus on sanitary fixtures, but on a product of a bodily evacuation, that is to say the urine, and its importance in Arabic as well as in Western medicine in the Middle Ages. Uroscopy, an art of establishing a diagnosis and a progonosis by examining the patient's urine collected in a special flak, was actually not born in Antiquity, but in Byzantium : the increasing part of the urine's examination in the clinical examination is due to Theophilus, a Byzantine author who's treatise on urines, called Peri ouron, influenced first byantine and arabic medicines : from the 10th century, the physician Isaac Israili (Ishāq al-Isrā'ilī) dedicated the first monography in arabic on that theme. Then, once hose two important works had been translated in latin in the 11th century, the Peri ouron of Theophilus and the Liber urinarum of Isaac Israeli, the Western medicine adopted their doctrine. This paper reminds the foundations of uroscopy in the Western and in the so called arabic medicine, and then, relying on textual but also iconographic documents, it aims to compare the respective place on urine's examination 
in those two areas, without forgetting the breaches that modalities of such an examination could open in the relationship between patients and practitioners.

Arabic Medicine - Clinical Examination - Diagnosis - Flask - Isaac - Medical Semiology - Medicine - Prognosis - Urines - Uroscopy - Theophilus - Urinal 
